Guillaume Castanet · Alain Delconte · Fabrice Lemoine

Loïc Mees · Gèrard Gréhan

\title{
Evaluation of temperature gradients within combusting droplets in linear stream using two colors laser-induced fluorescence
}

\begin{abstract}
The scope of this paper concerns the heating process of fuel droplets injected in a hot gaseous environment. The two colors laser-induced fluorescence technique allows measuring the temperature distribution within a droplet by scanning the droplet volume by a sufficiently small probe volume compared to the droplet volume itself. The temperature field is reconstructed using two approaches which have been compared. One is based on a geometrical optics model and the other is based on the 3D calculation of the internal excitation field within the droplet, using the generalized LorenzMie theory. Experimental results have been obtained on a combusting monodisperse ethanol droplet stream (diameter around $200 \mu \mathrm{m}$ ).
\end{abstract}

$\begin{array}{ll}\begin{array}{l}\text { List of symbols } \\ a_{I}, b_{I}\end{array} & \begin{array}{l}\text { Temperature sensitivity coefficients for the Ith } \\ \text { spectral band }\end{array} \\ C & \begin{array}{l}\text { Molecular concentration of the fluorescent } \\ \text { tracer }\end{array} \\ C_{\mathrm{F}} & \text { Friction coefficient } \\ C_{\mathrm{pl}} & \text { Liquid phase specific heat } \\ D & \text { Droplet diameter } \\ I_{\mathrm{f}} & \text { Fluorescence intensity } \\ K_{\mathrm{opt}} & \text { Optical constant } \\ K_{\mathrm{spec}} & \text { Spectroscopic constant } \\ R & \text { Droplet radius } \\ R e & \text { Droplet Reynolds number } \\ R_{\mathrm{f}} & \text { Fluorescence ratio }\end{array}$

G. Castanet $\cdot$ A. Delconte $\cdot$ F. Lemoine $(\triangle)$ LEMTA (CNRS UMR 7563), 2 , avenue de la Forêt de Haye, BP 160, 54504 Vandœuvre-lès-Nancy cedex, France E-mail: fabrice.lemoine@ensem.inpl-nancy.fr

L. Mees · G. Gréhan

CORIA (CNRS UMR 6614),

Avenue de l'Université, BP 12,

76801 Saint Etienne du Rouvray, France

$\begin{array}{ll}T & \text { Absolute temperature } \\ T_{\mathrm{I}} & \text { Injection temperature } \\ V_{\mathrm{I}} & \text { Injection velocity } \\ U_{\mathrm{S}} & \text { Maximum surface velocity } \\ (x, y, z) & \text { Spatial coordinates }\end{array}$

Greek symbols

$\beta \quad$ Temperature sensitivity coefficient

$\lambda_{1} \quad$ Liquid phase heat conductivity

$\mu_{1} \quad$ Liquid phase dynamic viscosity

$\mu_{\mathrm{g}} \quad$ Gas phase dynamic viscosity

\section{Subscripts \\ * $\quad$ Normalized quantities}

\section{Introduction}

The heating or cooling process of a fuel droplet entering a hot (or cold) gas environment is an important problem in several engineering applications such as spray combustion, freezing studies, and spray cooling of hot surfaces. The droplet heating can be investigated by measuring the droplet mean temperature, i.e., the temperature spatially averaged on the entire droplet volume. Further investigations require the characterization of the temperature within the droplet. Numerical studies (Chiang et al. 1992; Sazhin et al. 2002) have highlighted that fluid circulation within the droplets contributes noticeably to the within droplet heat transfer. One of the usual models of heat transfer within a droplet is the superposition of heat conduction with advection caused by the formation of a Hill vortex, due to the friction phenomena between the liquid droplet surface and the external air flow. 
Preliminary measurements of temperature fields of falling droplets have been reported by Winter and Melton (1990). The first transient measurements of the temperature distribution within a falling droplet have been obtained by Lu and Melton (2000). The droplets (about $500 \mu \mathrm{m}$ in diameter), initially at ambient temperature, were injected into a hot gaseous nitrogen ambient gas and the time to reach a homogeneous temperature field was investigated. In this experiment ( $\mathrm{Lu}$ and Melton 2000), the temperature has been measured by the exciplex fluorescence technique, reported in numerous references in the literature (e.g., Murray and Melton 1985 ; Wells and Melton 1990). Furthermore, the exciplex technique is very sensitive to quenching by oxygen, which constrains to operate under a neutral atmosphere. Consequently, the case of combusting droplets cannot be investigated by this technique.

The two colors laser-induced fluorescence (LIF) technique, using one single fluorescent tracer and two spectral bands of detection was developed to measure the mean droplet temperature. The main advantage of the two colors laser-induced fluorescence technique is the total independence of the measurement on the collection volume, i.e., the fraction of the droplet illuminated by the laser excitation, the local laser intensity and the tracer concentration. An extension of this technique has been proposed by Castanet et al. (2003), providing measurements of the temperature distribution within a droplet by scanning the droplet volume by a sufficiently small probe volume, compared to the droplet volume itself. The goal of the present paper is to discuss the reconstruction of the $3 \mathrm{D}$ temperature field using two approaches:

- One approach is based on a geometrical optics model developed in Castanet et al. (2003) using the 3D refraction law, which allows properly finding the position of the probe volume within the droplet, since there are deviations of the light at the air-liquid interface.

- The second approach is based on 3D calculation of the laser excitation field within the droplet using generalized Lorenz-Mie theory (GLMT) (Gouesbet et al. 1988; Gouesbet and Gréhan 2000).

Furthermore, the effect of temperature dependence of the liquid refractive index will be taken into account. The area of maximum intensity allows determining the measurement region within the droplet. After processing the experimental data, a temperature map in a meridian plane of the droplet can be constructed. Experimental results have been obtained on a combusting monodisperse ethanol droplet stream (diameter about $200 \mu \mathrm{m}$ ). Distribution of the temperature is determined at different times during combustion of the droplets; consequences of the within droplets fluid circulation on the heat transfer are clearly highlighted. Both approaches, one using geometrical optics and the other using GLMT, are compared.

\section{Two colors laser-induced applied to droplet temperature measurement}

Only the main outlines of the two colors laser-induced fluorescence technique are detailed in this section. Further technical details can be found in previous papers (Lavieille et al. 2001a; Castanet et al. 2003). The fuel (ethanol here) is previously seeded with a low concentration (a few $\mathrm{mg} / \mathrm{l}$ ) of rhodamine $\mathrm{B}$, which is an organic dye usually used as a fluorescent temperature sensor. Furthermore, the fluorescence of rhodamine $B$ can be easily induced by the green line $(\lambda=514.5 \mathrm{~nm})$ of the argon ion laser. It has been shown that its temperature sensitivity was strongly depending on the wavelength (Lavieille et al. 2001a). This technique allows removing the dependency in added tracer concentration, probe volume dimensions, laser intensity and optical layout. It requires two spectral bands for which the temperature sensitivity is highly different. This ratio of the fluorescence signal measured on the two spectral bands of detection appears only temperature dependent and is given by (Castanet et al. 2003):

$R_{\mathrm{f}}=\frac{I_{\mathrm{f} 1}}{I_{\mathrm{f} 2}}=\frac{K_{\mathrm{opt} 1}}{K_{\mathrm{opt} 2}} \frac{K_{\text {spec } 1} 1}{K_{\text {spec } 2}} \mathrm{e}^{\frac{a_{1}-a_{2}}{T}+\frac{b_{1}-b_{2}}{T^{2}}}$

where $K_{\mathrm{opt}}$ and $K_{\mathrm{spec}}$ are, respectively, optical and spectroscopic constants. $\left(a_{1}-a_{2}\right)$ and $\left(b_{1}-b_{2}\right)$ are temperature sensitivity coefficients, determined preliminarily in an initial calibration process (Lavieille et al. 2001a; Castanet et al. 2003).

The use of a single reference point, located close to the injector exit where the temperature is known, allows elimination of the optical and spectroscopic constants.

\section{Principles of the combustion experiment of monodisperse droplets}

A linear monodisperse droplet stream is generated by Rayleigh disintegration of a liquid jet (Lavieille et al. 2001a; Castanet et al. 2003) with the use of a mechanical vibration obtained by a piezoceramic excited by a square wave. The applied voltage on the piezoceramic depends on the desired position of the break-up zone and on the fuel physical properties related to the injection temperature. For a given frequency, the liquid jet breaks up into equally spaced and monosized droplets at the frequency of the forced mechanical vibration. The fuel can be pre-heated in the injector body by means of an external heated water circulation. The temperature of the fuel is measured exactly at the injection point with the use of a $\mathrm{K}$ type thermocouple. An electrically heated coil allows initiating the combustion just after the break-up zone of the liquid jet and creating a laminar flame. 

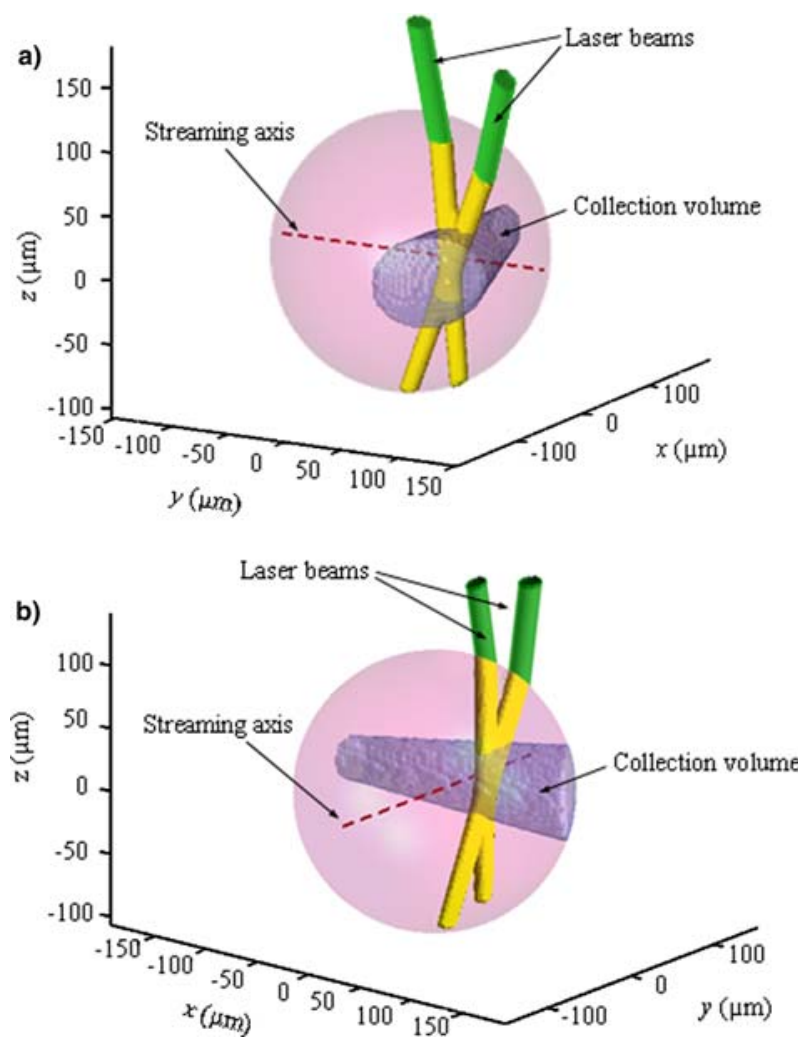

Fig. 1 Dimensions of the probe volume used for scanning the droplet $(x=20 \mu \mathrm{m}, y=60 \mu \mathrm{m}, z=0)$ (probe volume is the intersection between the collection volumes and the laser beams)

\section{Optical set-up}

The emission part of the optical set-up is based on LDV optics and is similar to that used in the paper of Castanet et al. (2003). The resulting excitation volume is an ellipsoid, i.e., the intersection zone of the two beams having the dimensions $20 \times 20 \times 90 \mu \mathrm{m}$ (Fig. 1). The same volume was used for fluorescence excitation and velocity measurements using a LDV chain, allowing accurate determination of the time elapsed from the injection point as well as the position of the probe volume in the droplet. The fluorescence signal is collected at a right angle; the effective collection volume is given by the intersection of the excitation volume with the collection optics solid angle. Taking into account the lensing effect of the air-droplet interface, the resulting probe volume dimensions are roughly $20 \times 20 \times 57 \mu \mathrm{m}$ (Fig. 1) when the intersecting point of the beam axis is located in the droplet center. The fluorescence signal is transmitted by an optical fiber to a set of beamsplitters and optical filters, which makes it possible to divide the fluorescence signal into the two specified spectral bands. Finally, the photons flux is converted into an electrical signal by means of photomultiplier tubes (Fig. 2) (Castanet et al. 2003).

\section{Principles of droplet temperature mapping and data reduction}

\subsection{Principles of droplet temperature mapping}

As a droplet crosses the laser excitation area in the stream direction, and if the probe volume is sufficiently small compared to the droplet, different zones of the droplet are successively illuminated by the laser and emit fluorescence, which can be processed with the two colors LIF technique. By shifting the probe volume in a perpendicular axis of the stream direction, it is possible to

Fig. 2 Block diagram of the experimental set-up

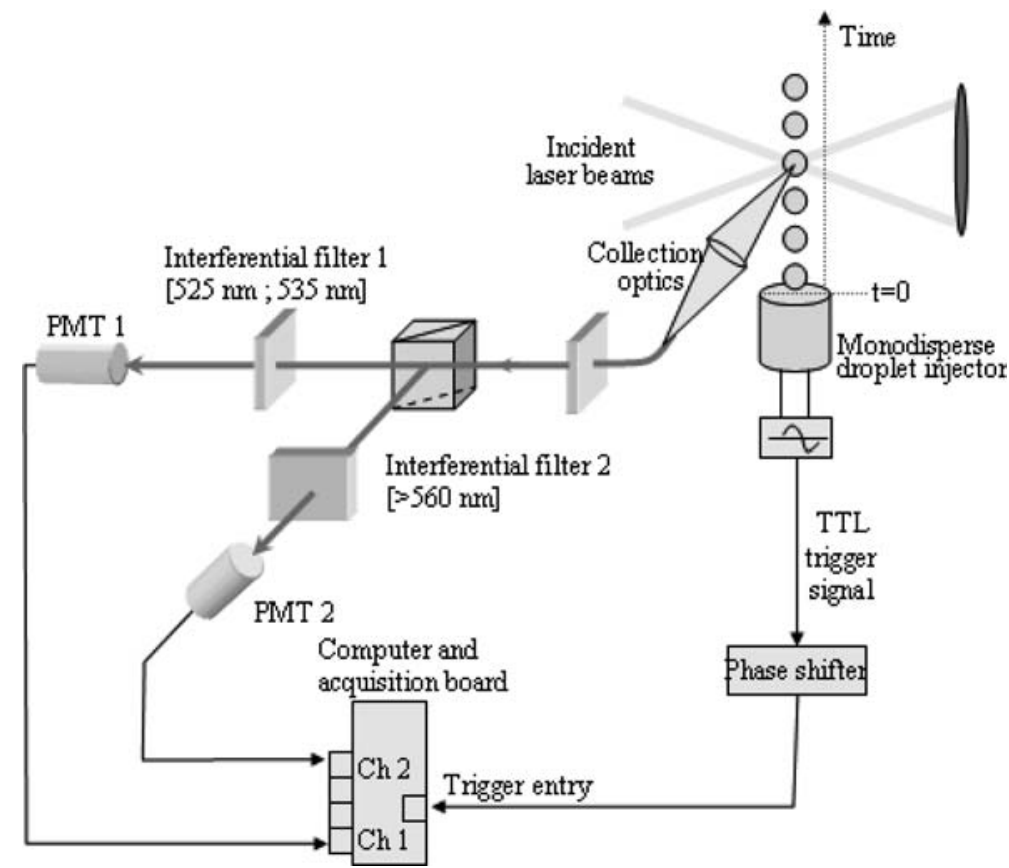




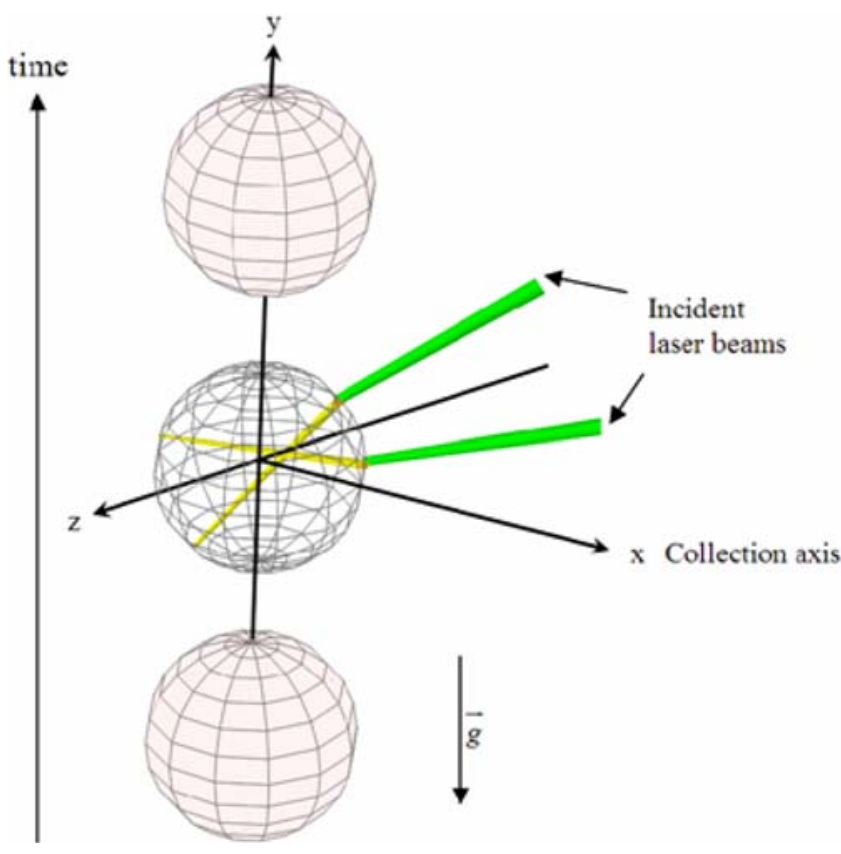

Fig. 3 Definition of the coordinates axis

scan different lines in the droplet. The different axes related to localization of the probe volume in the droplet are represented in Fig. 3. The droplet moves along the $y$ axis and the fluorescence is collected at a right angle on the $x$-axis. The bisecting line of the two laser beams is the direction of the $z$-axis, which is perpendicular to $x$ and $y$. The laser beams are therefore located in the $y, z$ plane, in absence of refraction due to the droplet interface. The position of the droplet in the fixed $(x, y, z)$ coordinate system is then determined by:

- The coordinate $y$, along the stream axis calculated by means of the measured droplet local velocity.

- The coordinate $x$, defining the distance between the stream axis and the measurement line along the $y$-axis.

- The coordinate $z$, which remains fixed at $z=0$ in the present case. The point $(x=0, y=0, z=0)$ is the droplet center.

- The droplet axis of symmetry $(x=0)$ is determined initially by the localization of the axis of symmetry of the fluorescence signal.

\subsection{Data acquisition and reduction}

The area of measurement can be localized by using a criterion based on the excitation intensity, since the contribution of a designated area to the total collected fluorescence signal is proportional to the local excitation intensity. The measurement area can be defined by the ensemble of the laser-illuminated points, which contribute mainly to the fluorescence emission. This area can be defined by the sum of two criteria:

- All the points with a local excitation energy higher than $x \%$ of the point of maximum energy of the excitation volume are retained. A value of $x$ ranging from $70 \%$ to $90 \%$ seems reasonable and practical.

- Only the points seen by the collection optics are retained.

The main features of the fluorescence signal acquisition strategy are described in Casatanet et al. (2003). The fluorescence signal distribution, corresponding to the scanning of the droplet in the streaming direction, is phase-locked and averaged on about 1,000 droplet transits, corresponding to a total acquisition time of less than 1s. The fluorescence ratio is calculated at each measurement point after completion of the averaging process and the temperature is determined by the relation between the fluorescence ratio and the temperature. To construct a temperature map, this process is repeated at different $x$ positions separated by $10-20 \mu \mathrm{m}$, depending on the droplet diameter. The lateral spread of the droplet trajectories is estimated to be less than $10 \mu \mathrm{m}$ by observing the droplet signal variations in the vicinity of the droplet border. This limits the distance of investigation from the injection point, since the turbulent motions due to the flame tends to increase the trajectory spread as the distance from the injection point is increased.

\subsection{Internal droplet excitation field}

Two strategies have been used in order to calculate the within droplet excitation field. The first one is based on geometrical optics applied to Gaussian laser beams while the second is based on GLMT (Gouesbet et al. 1988; Gouesbet and Gréhan 2000).

\subsection{Geometrical optics approach}

In a first step, the 3D refraction law at the droplet interface is applied to each of the two laser beam axes. The points of maximum excitation intensity are located in the intersecting zone of the two laser beams. The intensity distribution is given by the superposition of the intensity field of two individual Gaussian laser beams (Kogelnik and Li 1966; Lavieille et al. 2000). Two examples of meridian mapping of the excitation intensity field are presented in Fig. 4 with a logarithmic scale; one corresponds to a case where the laser beam's intersection is centered in the droplet, while the other corresponds to an off-centered position. In this calculation, a constant refractive index has been used, corresponding to the expected mean temperature range, between $30^{\circ} \mathrm{C}$ and $60^{\circ} \mathrm{C}$ (Lavieille et al. 2001b). This hypothesis will be discussed in Sect. 7.

\subsection{GLMT approach}

Calculation of the internal excitation field of the droplet is performed by GLMT. The original Lorenz-Mie theory 
Fig. 4 Equatorial map of the laser excitation intensity obtained by the GLMT and geometrical optics model. a GLMT, laser beam position: $x=0, y=0, z=0$. b Geometrical optics, laser beam position: $x=0, y=0, z=0$. c GLMT, laser beam position: $x=0$, $y=87.95 \mu \mathrm{m}, z=0$ d Geometrical optics, laser beam position : $x=0, y=87.95$ $\mu \mathrm{m}, z=0$
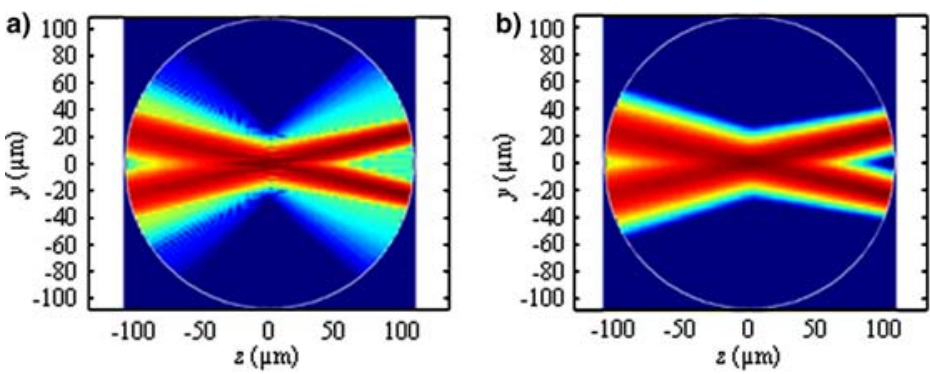

Intensity (A.U.)

\section{1
$10^{-1}$ \\ $10^{-2}$ \\ $10^{-1}$ \\ $10^{-4}$}
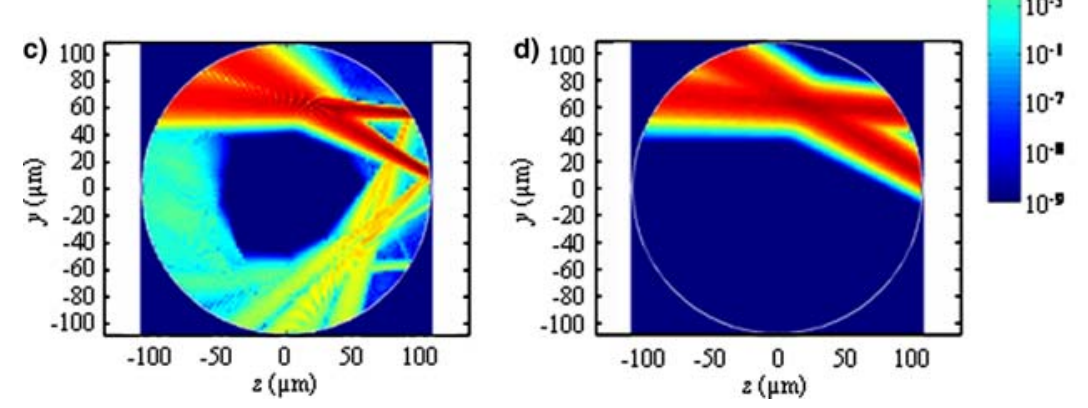

gives a rigorous solution to the problem of interaction between a plane wave and spherical particle. This theory has been extended to the case of partial illumination of the particle with a focused beam (Gouesbet et al. 1988). GLMT is able to quantify rigorously the scattered fields (far and near field), the internal fields and the radiation pressure forces from a regularly shaped particle illuminated by an arbitrary shaped beam. An overview of GLMT principles and applications can be found in Gouesbet and Gréhan (2000) and Mees et al. (2001). A computation code, based on GLMT, has been developed by the CORIA laboratory (Rouen), specially adapted for a LDV laser beam's system. The computation input parameters are:

- Liquid refractive index information (supposed constant as in the case of geometrical optics)

- Laser beam diameter in the focus point of the front lens of the LDV system

- Position $(x, y, z)$ of the beam axis intersection (when the droplet is not present)

- Wavelength of the laser radiation

- Beam angle (in the case of a LDV beam's system)

- Polarization status of the incident laser beams

- Information about the mesh
The code output is a 3D excitation field. After this calculation, the collection volume optics characteristics are included in the model, similarly to the case of the geometrical optics calculation. As for the geometrical optics, the case of an uniform refractive index will be considered. Two examples of an equatorial map of the excitation intensity field computed according to GLMT are presented in Fig. 4 with a logarithmic color scale in comparison with the calculations performed by geometrical optics already presented. The multiple reflections of the laser beams on the droplet interface are clearly visible and the area of measurement can be localized. One of the advantages of the GLMT approach is that the calculation is possible even if the beam's axis intersection point is located outside of the droplet under the geometrical optics point of view, since there is transfer of energy from the laser beams to the droplet. This makes possible, in principle, the determination of the liquid temperature nearer the droplet border as in the case of the geometrical optics model if a sufficient level of fluorescence signal is collected. An example of such a calculation is presented in Fig. 5 for a beam's axis intersection point located at $x=0, y=120 \mu \mathrm{m}, z=0$, the droplet border being
Fig. 5 Equatorial maps of the laser excitation intensity obtained by the GLMT. Case for which the intersecting point of the two laser beams is out of the droplet (coordinates of the intersection point: $x=0, y=120$ $\mu \mathrm{m}, z=0$ )
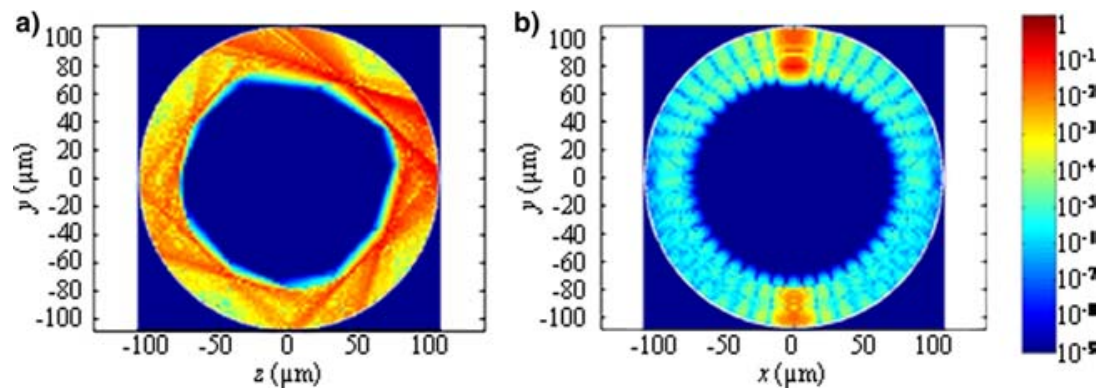
located at $y=108 \mu \mathrm{m}$; the localization of the measurement area, corresponding to the region of maximum excitation intensity, is well visible and seems clearly rejected inside the droplet .

5.6 Examples of processing by geometrical optics and GLMT

Calculation of the excitation field at different droplet positions has been performed with both geometrical optics and GLMT. According to the different axes of symmetry defined in Fig. 3, these calculations are performed only on a quarter of the droplet equatorial plane. Figures 6 and 7 respectively present the different positions where the excitation field has been calculated by geometrical optics and GLMT. Figures $6 \mathrm{a}$ and $7 \mathrm{a}$ give the points in the absolute axis system, while Figs. $6 \mathrm{~b}$ and $7 \mathrm{~b}$ give the same points positioned within the droplet according to internal intensity field calculation. Use of the criterion based on $90 \%$ of the point of maximum intensity results in a spot corresponding to the measurement area. One may notice that, in the particular case of GLMT, off droplets points can be calculated; however, the corresponding spots appear rejected inside the droplet due to the refraction of the laser beams and the multiple reflections at the droplet interface in the vicinity of the Brewster angle. After positioning of the measurement areas, the measured fluorescence ratio matching with the spots position is searched in the acquisition files, and the temperature corresponding to each spot is calculated using the temperature dependence law of the fluorescence ratio (Eq. 1). In the case of an overlap of two spots, the temperature corresponding to the intersecting zone is replaced by the mean value of the temperatures corresponding to the two spots.

\section{Temperature maps results}

The technique has been demonstrated on a combusting monodisperse ethanol droplet stream in the phase of the intense heating of the droplets. Monodisperse droplets of $216 \mu \mathrm{m}$ initial diameter are pre-heated in the injector body in order to make the inflammation easier. The injection conditions are $T_{\mathrm{I}}=35.3^{\circ} \mathrm{C}$ and $V_{\mathrm{I}}=9.8 \mathrm{~m} / \mathrm{s}$. The combustion is initiated $30 \mathrm{~mm}$ after the injector exit. The mean temperature evolution of droplets in a linear stream in a flame has been characterized by Lavieille et al. (2001b). A first phase corresponds to the intense heating of the droplet by forced convection due to the high background temperature. At the end of this phase, the droplet temperature attains the wet bulb temperature, inferior to the boiling temperature of the ethanol $\left(78^{\circ} \mathrm{C}\right)$. Due to the sharp temporal evolution of the mean droplet temperature in the heating phase, strong temperature gradients within the droplet are expected. The droplet velocity, measured by LDV is used for calculation of the time elapsed from the injector (Castanet et al. 2003). Temperature maps have been constructed at 50, 62,80 and $89 \mathrm{~mm}$ from the injection point, i.e., between the instants $t=5.3 \mathrm{~ms}$ and $t=9.6 \mathrm{~ms}, t=0$ being taken at the injector exit. Finally, the calculated diameter reduction, using the evaporation rate determined by Virepinte et al. (2000), is less than $5 \mu \mathrm{m}$ and has been therefore neglected in the data reduction process since the uncertainties linked to the positioning of the measurement points caused by the random motions of the droplet are approximately the same. The four temperature maps at the different instants, constructed by both the geometrical optics and GLMTapproaches, are shown in Fig. 8. In the light of the experimental temperature maps, its is clearly observed that the heat diffusion within the droplet does not occur radially as suggested by pure heat conduction. The heat seems to appear in the wake region, which is in qualitative agreement with the numerical simulations performed by Chiang et al. (1992). Furthermore, the temperatures maps determined with the GLMT appear very similar to those calculated by the geometrical optics: a satisfying cross-validation of the two approaches is noticed. The GLMT calculation is a rigorous approach, but is computationally time consuming since the computation of the internal excitation field within the droplet, for one position of the laser beams, takes several hours, whereas the
Fig. 6 Position of the points of maximum intensity within the droplet according to geometrical optics. a Position where the intensity field has been calculated (laser beam intersection points without the presence of droplet). b Spots corresponding to the calculated points positioned within the droplet, according to the $90 \%$ intensity criterion
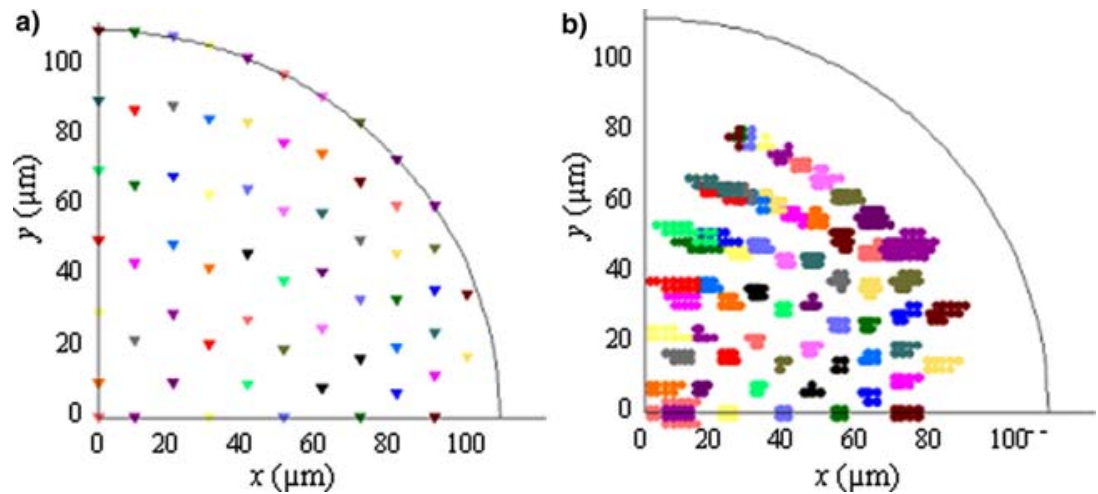
Fig. 7 Position of the points of maximum intensity within the droplet according to GLMT calculations. a Position where the intensity field has been calculated (laser beam presence of droplet). b Spots corresponding to the calculated points positioned within the droplet, according to the $90 \%$ intensity criterion intersection points without the

a) 120,
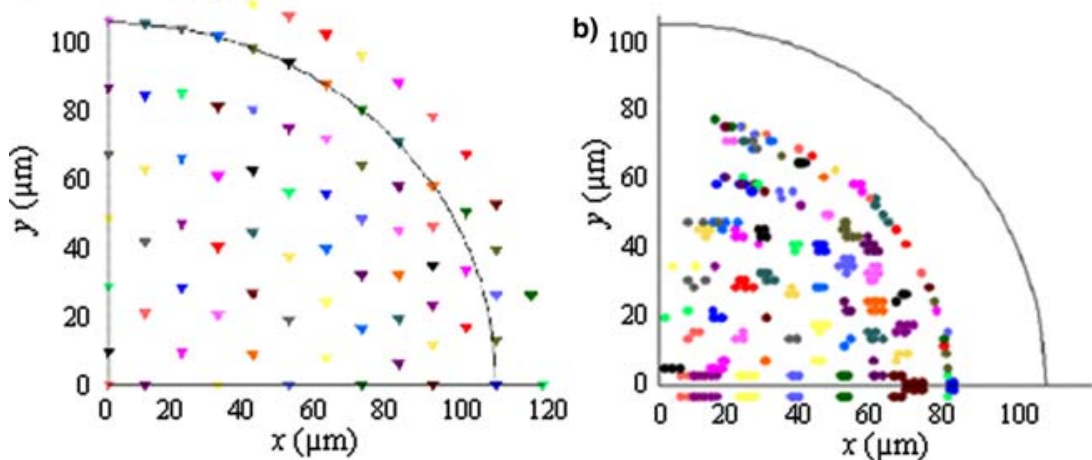

Fig. 8 Temperature maps calculated by both geometrical and GLMT approaches at different successive times of combustion (color code is in ${ }^{\circ} \mathrm{C}$, horizontal and vertical scales are in microns). The temperature maps are the results of a final interpolation, the spatial resolution being about $15 \%$ of the droplet diameter

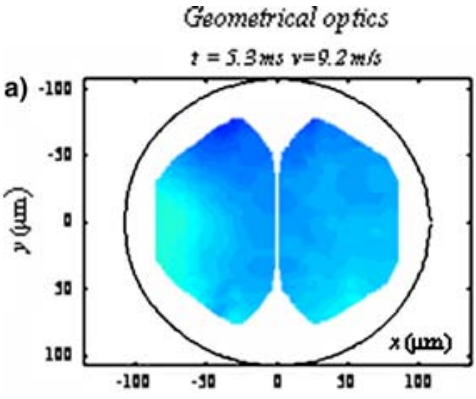

Generalized Lorenz-Mie Theory (GLMT)
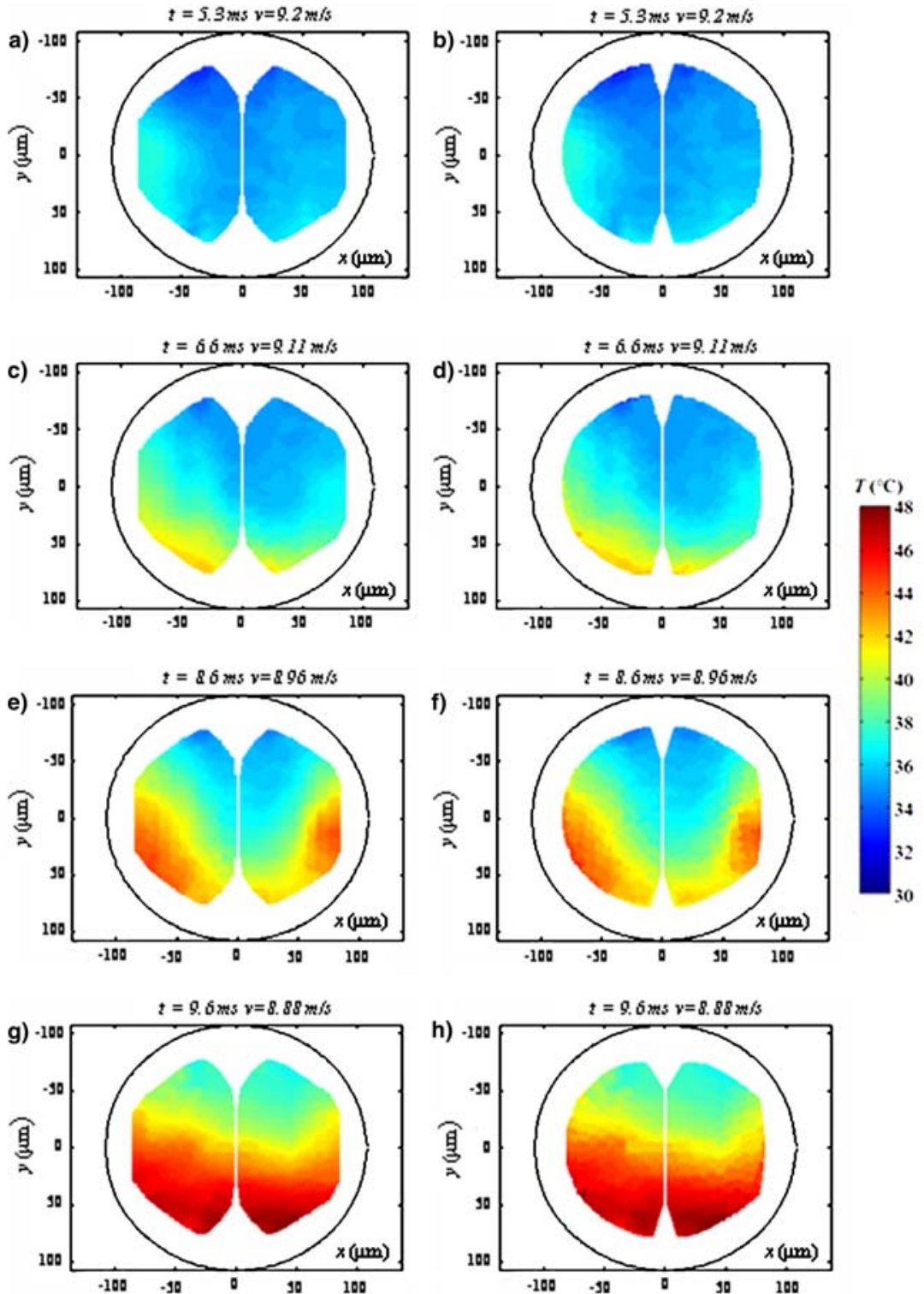

geometrical optics approach takes only a few seconds. It may also be noticed that the area covered by the GLMT calculations is a little larger than the area de- scribed by geometrical optics. However, in this optical configuration, a significant zone remains inaccessible to the experiment. 


\section{Influence of the refractive index gradients}

Both data reduction procedures (geometrical optics and GLMT) are based on the hypothesis of a constant refractive index within the droplet. In order to test the validity of this assumption, a numerical calculation of the internal temperature field has been carried out in order to obtain a refractive index map, knowing the temperature dependence of fuel refractive index. The use of a calculated temperature map has been preferred to the experimental one, since the temperature can be determined throughout the droplet volume, including the zones inaccessible to the experiment. Furthermore, smoothed isothermal lines can be obtained, which makes the further calculations more reliable.

\subsection{Numerical simulation principles}

The droplet heating is the result of the heat release from the combusting fuel vapor, creating a hot gas environment surrounding the droplets. Two combined mechanisms are generally involved to explain the heat transfer inside the moving liquid droplets: pure heat conduction and the advection by the internal motions caused by the friction phenomena between the liquid droplet surface and the external air flow. The general assumptions of the heating model are:

- The temperature distribution along the droplet surface is uniformly distributed and varying in time.

- The droplet surface temperature at each time step is re-adjusted following the algorithm proposed by Abramzon and Sirignano (1989).

- The instantaneous velocity field inside the streaming droplet is approximated by the Hill spherical vortex solution (Abramzon and Sirignano 1989; Clift et al. 1978).

As a consequence, the within droplet temperature field can be calculated by solving the dimensionless heat transfer equation, neglecting the viscous dissipation:

$\frac{\partial T^{*}}{\partial t^{*}}+\overrightarrow{V^{*}} \cdot \overrightarrow{\operatorname{grad}^{*}} T^{*}=\frac{1}{P e} \Delta^{*} T^{*}$.
In this dimensionless equation, the spatial coordinates are reduced by the droplet radius.

The temperature $T$ is reduced by the surface temperature $T_{\mathrm{S}}$ and the velocity components by the maximum velocity $U_{\mathrm{S}}$ at the droplet surface.

The dimensionless time is defined as follows:

$t^{*}=\frac{U_{\mathrm{s}}}{R} t$

Finally, the Peclet number $P e$ can be written :

$\mathrm{P} e=\frac{\rho C_{\mathrm{p} l} R}{\lambda_{l}} U_{\mathrm{S}}$.

The maximum surface velocity is determined by the friction component of the droplet drag force, and according to Sirignano (1999), in the case of a moving isolated droplet, can be expressed by:

$U_{\mathrm{S}}=\frac{1}{6 \pi}\left(V_{\infty}-V\right)\left(\frac{\mu_{\mathrm{g}}}{\mu_{l}}\right) \operatorname{Re} C_{\mathrm{F}}$,

where $V_{\infty}$ is neglected in the present case, since the droplet stream is injected in a quiescent atmosphere. The physical properties of the gas phase and the droplet Reynolds number $R e$ are calculated at the reference state according to the " $1 / 3$ rule" (Hubbard et al. 1975).

The calculation of the droplet temperature field is performed under a quasi-steady assumption: at each time step $(0.1 \mathrm{~ms})$, the Peclet number is updated as well as the physical properties of the gas phase.

The friction coefficient $C_{\mathrm{F}}$ is adjusted by comparison with the experimental temperature map at $t=9.6 \mathrm{~ms}$ (last temperature map position), in order to obtain better agreement between the experiment and the calculation. In order to optimize this adjustment, the averaging effect of the measuring volume has been applied on the calculated temperature map according to:

$$
I_{\text {averaged }, I}=\frac{\int_{V} I(x, y, z) \mathrm{e}^{\frac{a_{i}}{T(x, y, z)^{2}}-\frac{b_{i}}{T(x, y, z)}} \mathrm{d} x \mathrm{~d} y \mathrm{~d} z}{\int_{V} I(x, y, z) \mathrm{d} x \mathrm{~d} y \mathrm{~d} z}, \quad I=1 \text { or } 2,
$$

Fig. 9 Comparison with the experimental temperature map and the numerical simulation at $t=9.6 \mathrm{~ms}$. The space averaging effect of the optical system has been taken into account in the numerical temperature map
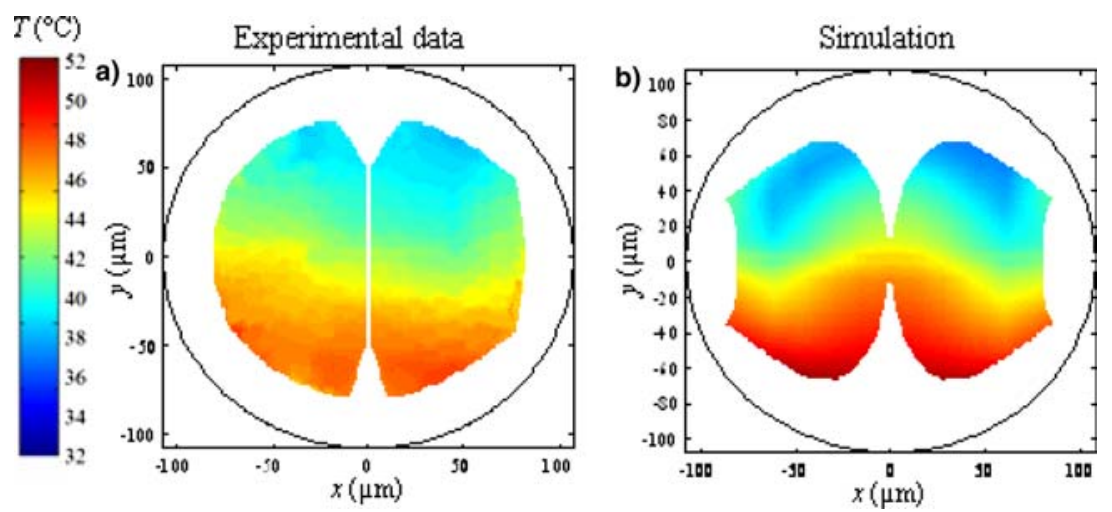
where $I(x, y, z)$ and $T(x, y, z)$ represent, respectively, the excitation intensity and the calculated temperature at the position $(x, y, z)$ and $V$ is the measuring volume. The index $I$ correspond to the first or second spectral band of detection.

Comparison between the experiments and calculations, after adjustment of the friction coefficient, is reported in Fig. 9 for the time $t=9.6 \mathrm{~ms}$. Figure 9a and b represents the comparison between the experimental temperature map constructed by GLMT and the calculated temperature map after applying the averaging effect of the measuring volume. The agreement between the numerical simulations and the experiment seems to be good. Thus, the calculation results will be used to study the effect of the variable refractive index within the droplet. Figure 10 presents the results of the numerical simulation without any averaging effect, and the calculation of the isothermal lines for steps of $1{ }^{\circ} \mathrm{C}$.

\subsection{Study of the refractive index gradients effect}

The isothermal lines, corresponding also to the lines of constant refractive index, are calculated in steps of $1{ }^{\circ} \mathrm{C}$, the first isothermal line being the droplet surface. Modification of the measuring area can be calculated only using the geometrical optics approach, since GLMT calculations can be carried out only under spherical symmetry assumptions. In this calculation, only the axis of the laser beams will be considered, but it has been observed that the maximum excitation energy is concentrated in the vicinity of this point.

The interaction of the two laser beams with each isothermal surface (equally spaced of $1^{\circ} \mathrm{C}$ ) is calculated by applying the $3 \mathrm{D}$ refraction law. The intersection, if existing, of the two laser beams corresponds to the measurement point. If there is no intersection, the average position between the closest points of the two beams is considered and it can be observed that the

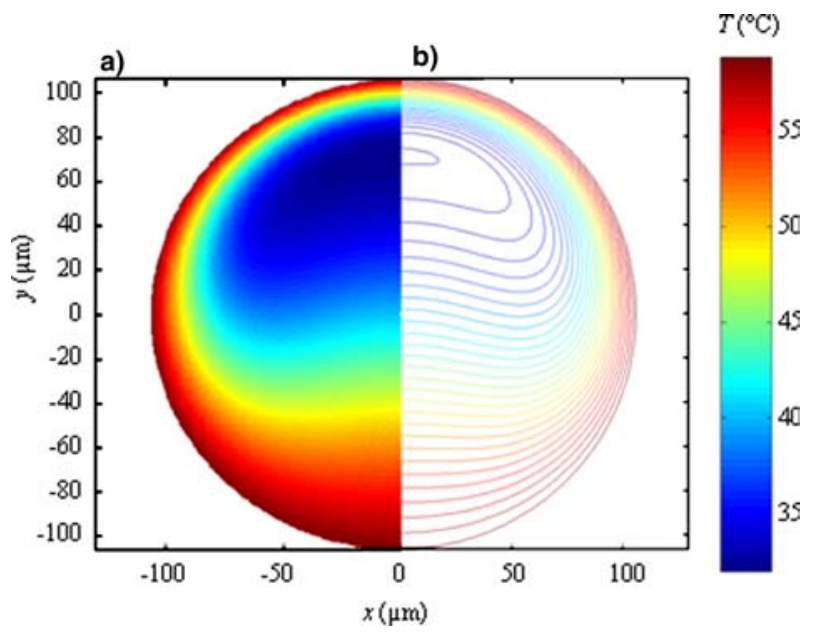

Fig. 10 Temperature map and the numerical simulation at $t=9.6 \mathrm{~ms}$, and calculation of the isothermal lines by steps of $1^{\circ} \mathrm{C}$

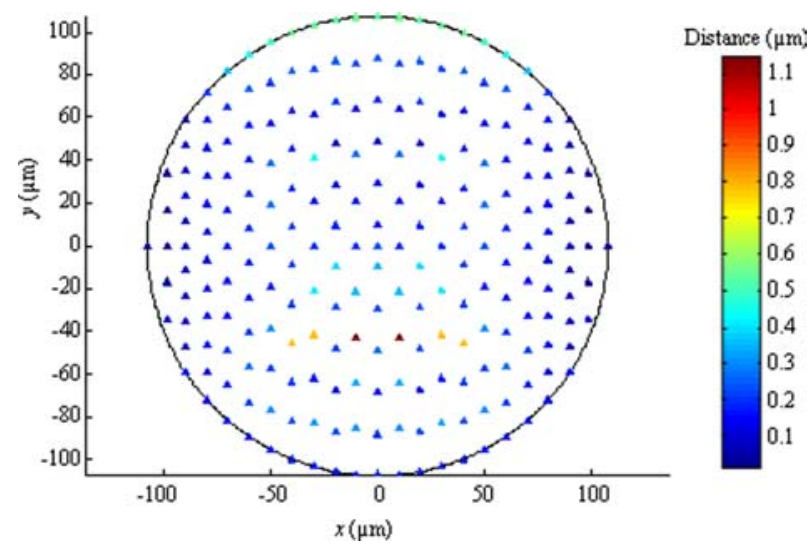

Fig. 11 Shift between the measurement points obtained by both calculation procedures, using a constant or a variable refractive index, according to the numerical simulation. The shift is calculated at $t=9.6 \mathrm{~ms}$

maximum distance between the closest points of the two beams never exceeds $10 \mu \mathrm{m}$.

The same calculation is performed with a constant refractive index corresponding to the droplet mean temperature. The positions of the measurements points obtained by both calculation procedures (constant or variable refractive index) are compared in Fig. 11, where the shift between the two calculations is represented. The calculation grid is similar than the grid used for the GLMT calculation. In the light of Fig. 11, it can be clearly concluded that the refractive index gradients cause a very moderate shift of the measurement points positions, less than $1 \mu \mathrm{m}$ in the worst cases, which is clearly in the position of uncertainty.

\section{Conclusions}

Parts of the temperature map within a combusting droplet can be measured with the use of the two colors LIF technique, by scanning the droplet with a sufficiently small probe volume, compared to the droplet size. The positioning of the measurement points within the droplet is a delicate operation and requires the calculation of the within droplet excitation field. Two kinds of approaches have been used:

- A simplified geometrical optics calculation, based on refraction of Gaussian laser beams (which requires a very short computation time)

- A rigorous approach using GLMT (more computationally time consuming)

The geometrical optics calculations are well validated by the GLMT, and a slightly larger area of the equatorial plane of the droplet is covered with the use of the GLMT. The examination of the temperature maps reveals the presence of noticeable temperature gradients within the droplets. The careful observation of this temperature distribution suggests that the transport of 
heat is connected with internal fluid motions combined with pure heat conduction. Strong temperature gradients in the vicinity of the droplet surface are also suspected, since the surface temperature is in the vicinity of $60^{\circ} \mathrm{C}$ (Lavieille et al. 2001b), but cannot be resolved by the present technique.

Comparison of the experimental temperature fields with preliminary computation based on resolution of the heat transfer equation combined with the velocity field given by the Hill vortex solution is encouraging. With the results of this computation, the effect of the propagation of the beams in a nonconstant refractive index medium has been examined. It has been shown that the difference with a constant refractive index assumption was negligible. In a next step, a parametric study will be carried out in order to compare the experimental data with the numerical simulations and to adjust the vortex characteristic parameters for different aerothermal injection conditions.

Acknowledgment This work is supported by the European Community in the framework of the MUSCLES contract, Growth project GRD1-2001-40198.

\section{References}

Abramzon B, Sirignano WA (1989) Droplet vaporization model for spray combustion calculations. Int $\mathbf{J}$ Heat Mass Tran 32(6): 1605

Castanet G, Lavieille P, Lebouché M, Lemoine F (2003) Measurement of the temperature distribution within monodisperse combusting droplets in linear stream using two colors laserinduced fluorescence. Exp Fluids 35:563-571

Chiang CH, Raju MS, Sirignano WA (1992) Numerical analysis of convecting, vaporizing fuel droplet with variable properties. Int J Heat Mass Trans 35(5):1307-1324

Clift R, Grace JR, Weber ME (1978) Bubbles, drops, and particles. Academic, New York

Gouesbet G, Gréhan G (2000) Generalized Lorenz-Mie theories, from past to future. Atomization Spray 10(3-5):277-333
Gouesbet G, Maheu B, Gréhan G (1988) Light scattering from a sphere arbitrarily located in a Gaussian beam, using a Bromwich formulation. J Opt Soc Am A5:1427-1443

Hubbard GL, Denny VE, Mills AF (1975) Droplet evaporation: effects of transients and variable properties. Int $\mathrm{J}$ Heat Mass Transf 18:1003-1008

Kogelnik H, Li T (1966) Laser beams and resonators. Appl Opt 5(10):1550-1566

Lavieille P, Lemoine F, Lavergne G, Lebouché M (2001a) Evaporating and combusting droplet temperature measurements using two-color laser-induced fluorescence. Exp Fluids 31(1):45-55

Lavieille P, Lemoine F, Lebouché M, Lavergne G (2001b) Mesure de la temperature de gouttelettes en combustion par fluorescence induite par laser a deux couleurs: resultats preliminaires et perspectives. Comptes rendus de l'Academie des Sciences Serie IIb Mecanique 329(8):557-564

Lavieille P, Lemoine F, Lebouché M (2002) Investigation on temperature of evaporating droplets in linear stream using twocolor laser-induced fluorescence. Combust Sci Technol 174(4):117-142

Lavielle P, Lemoine F, Lavergne G, Virepinte JF, Lebouché M (2000) Temperature measurements on droplets in monodisperse stream using laser-induced fluorescence. Exp Fluids 29:429

Lu QZ, Melton LA (2000) Measurement of transient temperature field within a falling droplet. AIAA J 38(1):95-101

Mees L, Gouesbet G, Gréhan G (2001) Interaction between femtosecond pulses and a spherical microcavity: internal fields. Opt Commun 199:33-38

Murray AM, Melton LA (1985) Fluorescence methods for determination of temperature in fuel sprays. Appl Opt 24(17):27832787

Sazhin SS, Dombrovsky LA, Krutitski P, Sazhina EM, Heikal MR (2002) Analytical and numerical modelling of convective and radiative heating of fuel droplets in diesel engines. In: Proceedings of the international heat transfer conference, 18-23 August 2002, Grenoble, France (on CD)

Sirignano WA (1999) Fluid dynamics and transport of droplets and sprays. Cambridge University Press, Cambridge

Virepinte JF, Biscos Y, Lavergne G, Magre P, Collin G (2000) A rectilinear droplet stream in combustion: droplet and gas phase properties. Combust Sci Technol 150(1-6):143-159

Wells MR, Melton LA (1990) Temperature measurements of falling droplets. T ASME 112:1008-1013

Winter M, Melton LA (1990) Measurement of internal circulation in droplet using laser-induced fluorescence. Appl Opt 29(31):4574-4577 